\title{
L'argument axiologique dans la Philosophie de Dieu de St Augustin
}

L'objet central de l'interêt de l'évêque d'Hippone a été Dieu: "Connaître Dieu et l'âme: voilà ce que je désire. Et rien de plus? Rien absolument" ". Dieu, comne Etre suprême et Bien suprême, a été la lentille, où se contraient tous les interêts et les problèmes. Toutes choses dérivent de Dieu et toutes — par l'amour - retournent à Dieu. Il est "la vie des âmes, la vie des vies" 2 . Il accomplit notre contingence sous l'aspect ontologique, épistémologique et éthique. Dieu est la Vérité, à laquelle chaque homme aspire, et le Bien, qui peut lui donner un bonheur plénier.

La théodicée augustinienne contient quelques arguments, au moins implicite, pour prouver l'existence de Dieu: idéologique, cosmologique, téléologique, éudémologique et axiologique ${ }^{3}$. L'argument axiologique (de la hiérarchie des valeurs) est de caractère rationnel, parce qu'il suppose les premiers principes logico-ontologiques. A propos de cet argument, parlent de façon générale: J. Mausbach, J. Hessen, M. Grabmann ${ }^{4}$. Celui qui a le plus écrit c'est Fr. von Rintelen, d'après qui saint Augustin conclut l'existence de Souverain Bien de deux marières: la né-

1 Soliloquia I, 2, 7 PL. 32, 872. Bibliothèque Augustinienne. Oeuvres de saint Augustin, t. V. Trad. par R. Jolivet, P. de Labriolle, F.-J. Thonnard, Paris 1955, Desclée de Brouwer, 261-262. Nous citerons les oeuvres de saint Augustin d'après: Bibliothèque Augustinienne, BA, Pour les oeuvres, qui n'ont pas été publiées dans cette édition, nous citerons d'après: Migne, Patrologia Latina, t. 32-47, Paris 1861-62.

2 Confessiones 3, 6, 10 BA, t. XIII. Texte de l'édition de M. Skutella, introduction et notes par A. Solignac, traduction de $\mathrm{E}$. Théhorel et G. Bouissou, Paris 1962, 383, PL. 32, 687. $20 \mathrm{ss}$

3 Voir: F. CAYRÉ, Initiation à la philosophie de saint Augustin, Paris 1947,

4 J. Mausbach, lie Ethik des heiligen Augustinus, Freiburg 1909, I, 92 ss; J. Hessen, Augustins Metaphysik der Erkenntnis, Berlin 1931, 150-152; M. Grabmann, Iie Grundlagen des heiligen Augustinus über Seele und Gott, Köln 1929,89 . 
gative et la positive ${ }^{5}$. La manière négative se trouve dans les Confessions, où on conciut à son existence par les "défauts" inhérents à la nature de toutes les choses visibles ${ }^{6}$. Il faut dire, que Fr. von Rintelen ne distingue pas les arguments cosmologiques et axiologiques. Dans les Confessions $(\mathrm{X}, 6)$ il y a seulement l'argument cosmologique, qui parie de l'existen. ce de Dieu en tant que l'Artifex du monde. L'aspect axiologique y est relégué au second plan ${ }^{7}$. Sur l'argument axiologique G. Esser a aussı écrit, mais il cite seulement les textes de saint Augustin et ne les interprète pas ${ }^{8}$.

Le texte classique de l'argument axiologique chez saint Augustin est le suivant: "Tu n'aimes certes que le bien. Car bonne est la terre avec ses hautes montagnes, ses collines mesurées, ses planes campagnes; bon, le domaine agréable et fertile; bonne la maison vaste et claire, aux proportions harmonieuses; bon, le corps animal doué de vie; bon, l'air tempéré et salubre; bonne, la nourriture savoureuse et saine; bonne, la santé sans souffrances ni fatigues; bon, le visage de l'homme, harmonieux, enjoué, éclatant de fraîcheur; bon, le coeur de l'ami, da douceur de partager les mêmes sentiments, la foi de l'amitié; bon, l'homme juste $[\ldots]$. Ceci est bien, cela est bien. Supprime le ceci et le cela, et vois, si tu peux, le bien même: alors tu verras Dieu, qui ne tient pas sa bonté d'un autre bien, mais est la bonté de tuot bien. En effet, parmi tous ces biens - ceux que j'ai rappelés ou d'autres que l'on voit ou que l'on imagine- nous ne pourrions pas dire que l'un est meilleur que l'autre, lorsque nous pensons juste, si n'était imprimée en nous la notion du bien même, règle de nos approbations, règle de nos préférences [...]. Il n'y aurait donc pas de biens changeants, s'il n'y avait pas un bien immuable. Voilà pourquoi, lorsqu'on entend parler de tel ou tel bien, lequel, vu d'un certain biais, ne mérite pas le nom de bien: si on arrive à faire abstraction de ces biens, qui ne sont tels que par participation, pour voir le bien dont ils participent (car ce bien, on en a l'intelligence, au moment même où l'on entend parler de tel ou tel bien); si donc on

5 Fr. von RINTELEN, Der Wertgedanke in der europäischen Geistesentwicklung, Halle 1923, 163 ss.; Deus bonum omnis boni, dans: Aurelius Augustinus, Köln 1930, 203 ss.

6 Voir: Confessiones 10, 6, 8-9, BA, t. XIV, 152-156, PL. 32, 781-782.

7 Voir: Ibid. 11, 4, 6 BA, t. XIV, 280, PL. 32, 811.

8 G. Esser, The Augustinian Proof for God's Existence and the Thomistic fourth Way, "Proceedings of American Catholic Philosophical Association", 28, 1954, 196-199. 
arrive, faisant abstraction de ces biens, à voir le bien par lui-même, on voit Dieu. Et si l'on s'attache à dui par amour, du même coup on trouve le bonheur" 9 .

Sur l'éxistence du Bien Suprême l'évêque d'Hippone a écrit encore ailleurs ${ }^{10}$. Quoique Augustin ne formule jamais l'argument axiologique expressis verbis, il est vrai que cet argument nous pouvons le formuler à partir les textes augustiniens. Au commencement saint Augustin exprime avec force l'optimisme axiologique: "Et que toute nature, en tant que nature, est un bien" ${ }^{11}$. Le bien ontologique est universel. Mais il est hiérarchiquement disposé. Dans le monde nous voyons la trés riche hiérarchie des valeurs; les unes sont ontologiquement des biens, seulement grâce à l'existence du réel, comme les corps célestes (les étoiles, le soleil', la lune) et les êtres inorganiques (p. ex. les minéraux); les autres ont déjà le bien de la vie, comme les plantes; ont un meilleur bien les animaux parce qu'ils ont la connaissance sensitive et l'instinct; les hommes ont le meilleur bien du monde comme êtres raisonnables.

L'axiologie augustinienne a très expressément le caractère humain. L'authentique humanisme doit affirmer toutes les valeurs, qui rendent possible le développement de la personnalité humaine sous tous ses aspects: matériel, biologique et spirituel. Saint Augustin sait, que l'homme est situé au croisement du monde matériel et spirituel. C'est pourquoi chaque homme, pour l'harmonie du développement de sa personnalité, a autant besoin des valeurs matérielles que des valeurs spirituelles. Au début, la philosophie n'est pas la recherche du bien suprême pour les plantes ou pour les animaux, mais pour les hommes ${ }^{12}$. La personnalité humaine est le point central de tout bien; mais elle n'est pas sa source.

Saint Augustin a divisé toutes les valeurs en trois groupes. Le pre-

9 De Trinitate 8, 3, 4-5 BA, t. XVI. Texte de l'édition Bénédictine, traduction par P. Agaësse, Paris 1955, 33-37, PL. 42, 949-950.

10 "Dicitur enim bonus homo, et bonus ager, et bona domus, et bonum animal, et bona arbor, et bonum corpus, et bona anima [...] Est' bonum simplex, ipsum bonum quo cuncta sunt bona, ipsum bonum ex quo cuncta sunt bona" Enar. in ps. 26, 8 PL. 36, 203. Voir: Enar. in ps. 134, 4 PL. 37, 1740; Epist. 118, 3, 13, PL. 33, 438.

11 De natura boni 1, 1 BA, t. I. Texte, traduction et notes par B. RolandGosselin, Paris 1936, 193, PL. 42, 557.

12 "Summun bonum hoc est tuum bonum [...] Pecori quid bonum est [...] nisi adimplere ventrem [...] dormire, gestire, vivere, sanum esse, generare [...] Tale bonum tu quaeris?". (Enar. in ps. 102, 8 PL. 37, 1321). Voir: De civ. Dei 19, 3 PL. 41, 625'; P. DelHAYE, "Saint Augustin et les valeurs humaines": Mélanges des sciences religieuses: 12 (1955) 121-130. 
mier groupe contient les valeurs de la vie et de la sensibilité (p. ex. la santé, la nourriture, la volupté), qui sont au service avant tout du corps humain ${ }^{13}$. Le second groupe, celui des valeurs spirituelles, qu'il faut diviser en valeurs esthétiques (un beau visage, le rythme de la poésie, la structure de l'édifice), valeurs intellectuelles (la science, l'érudition, la sagesse), et valeurs éthiques (l'amitié, le bon coeur, la vertu). La valeur suprême c'est l'amour, étant au croisement de la nature et de grâce. Le troisième groupe ce sont les valeurs extérieures (bona externa) à la personnalité humaine, p. ex. l'argent, la gloire, etc. Cette gradation des valeurs, était déjà connue de la philosophie grecque, dont s'inspire Origène, chef de l'école catéchetique d'Alexandrie ${ }^{14}$.

La gradation des valeurs, précisée dans le De Trinitate, contenait non seulement le bien moral, mais toutes les valeurs. C'est pourquoi il faut dire, que l'argument axiologique selon saint Augustin se fonde sur chaque valeur. Quand il parle de la hiérarchie des valeurs, c'est prendre en considération des traits caractéristiques tels que: la durabilité, l'autonomie ontique, la capacité de satisfaction du désir de félicité des hommes. Les valeurs spirituelles sont hiérarchiquement plus grandes que les valeurs matérielles et sensibles, c'est pourquoi elles sont plus durables et plus universelles.

L'authentique humanisme axiologique chez saint Augustin se lie avec le théocentrisme, parce que la structure graduée des valeurs démontre l'existence de la Valeur Suprême. Cette méthode de la théodicée augustinienne, se manifeste de façon synthétique dans les paroles des Confessions: "Je dépasserai donc encore cette force de ma nature, m'élevant par d e g r és jusqu'à celui qui m'a fait" ${ }^{15}$. La hiérarchie des valeurs exprime leur progressif accroissement. Nous voyons des valeurs moins bonnes ou meilleures, mais toujours hétéronomes: limitées et fragiles. Le couronnement naturel de toutes les valeurs visibles, hiérarchiquement órdonnées, est le Bien Suprême. Il est entièrement autonome dans son existence et illimité dans sa perfection.

Saint Augustin a écrit "Supprime le ceci et le cela, et vo is, si tu peux, le bien même, alors tu verras Dieu". Dieu est "vu" dans les va-

13 Voir: De civ. Dei 8, 8 PL. 41, 232-233; 19, 3 PL. 41, 625-626.

14 "Tres esse species boni : unam ergo animam, aliam ergo conpora, tentium extrinsecus". (Comm. in epist. ad Rom. 3, 1 PG. 14, 927). Voir: Selecta in psalmos 4 PG. 12, 1151-1155.

15 Confessiones 10, 8, 12 BA, t. XIV, 161, PL. $22,784$. 
leurs du monde? La terminologie - vide, videbis, visio - suggère que nous connaissons Dieu immédiatement. De cette manière a interpreté le texte J. Hersen ${ }^{16}$. Probablement cette interprétation n'est-elle pas exacte, parce que chez saint Augustin le terme "visio" a un caractère analogique. Visio c'est non seulement la connaissance immédiate, mais aussi la connaissance certaine. En deuxième lieu "visio" peut signifier l'abrégé du raisonnement. Augustin, dans le commentaire sur la Genèse, a discerné trois sortes de visions: une vision corporelle, une vision spirituelle et une vision intellectuelle ${ }^{17}$. Le commentaire sur le psaume I49 distingue deux sortes de visions ${ }^{18}$. L'une d'elles se réalise dans la vie temporelle grâce à la foi (per fidem), et l'autre se réalisera dans la vie future chez les élus (facie ad faciem). Il est très caractéristique, que chez saint Augustin la connaissance de Dieu par la foi, est appelée "visio". D'ailleurs dans la lettre I 47 il a écrit, que la connaissance immédiate de Dieu est actuellement impossible ${ }^{19}$. Finalement il faut dire, que les mots - visio, vide - signifient seulement la connaissance intuitive ${ }^{20}$. Elle se distingue de la connaissance spéculativo-discursive, mais n'est pas la contemplation inmédiate de Dieu. Le Bien Suprême est "vu" à travers les valeurs de la même manière que la Cause Suprême est "vue" par les relations causales du monde.

Lors de l'interprétation du texte du livre VIII de De Trinitate les mots suivants sont difficiles à comprendre: "Nous ne pourrions pas dire que l'un est meilleur que l'autre, lorsque nous pensons juste, si n'était imprimée en nous la notion du bien même, règle de nos approbations, règle de nos préférences" ${ }^{21}$. Avons-nous en nous la conception innée de Dieu comme Bien Suprême? La réponse positive peut être assimilée à la théorie de l'innéité. Probablement saint Augustin a accepté

16 J. Hessen, Augustins Metaphysik der Erkenntnis, Berlin 1931, passim.

17 Gen. ad litt. 12, 6, 15 PL. 34, 458; R. AlLERs, Illuminations et vérités éternelles: une étude sur l'a priori augustinien, dans: Augustinus Magister, I, Paris 1954, 477-490.

18 "Est quaedam visio huius temporis, erit altera visio futuri temporis, visio quae modo est, per fidem est; visio, quae futura est, per speciem est". (Enar. in ps. 149, 4 PL. 37, 1951). Voir: Epist. 148, 9 PL. 33, 626.

19 Voir: Epist. 147 PL. 33, 596-622. Cette lettre a été écrit par saint Augustin apprès l'an 411 .

20 Voir: W. FALKENHAHN, Augustin Illuminationstheorie im Lichte der jüngsten Forschungen, Köln 1948; C. E. ScHÜTzINGER, "Die Augustinische Erkenntnislehre im Lichte neuer Forschung": Recherches Augustiniennes 2 (1962) 177-203; U. AlvaREz, "El problema de la abstracción en S. Agustín. Interpretaciones históricas": La ciudad de Dios 173 (1960) 397-443.

21 De Trinitate 8, 3, 4 BA, t. XVI, 33, PL. $42,949$. 
l'apriorisme modéré, mais il n'a pas accepté l'extrémisme nativiste. De cette manière interprètent l'épistémologie augustinienne: Cayré, Sciacca et Piemontese. Pour F. Cayré l'apriorisme de l'évêque d'Hippone est comme une sorte d'intuition intellectuelle ${ }^{22}$. M. F. Sciacca a vu chez saint Augustin avant tout l'intérieurisme de la vérité ${ }^{23}$. Selon Piemontese l'épistémologie augustinienne a été basée sur la connaissance réflexive, la conscience et l'introspection ${ }^{24}$.

Après tout, il faut dire, que l'évêque d'Hippone n'a pas accepté la conception innée du Bien Suprême. Faut-il interpréter le passage précité comme l'intuition rationelle, analogue à celle de saint Thomas d'Aquin (intellectus principiorum), et entièrement différente de l'intuition emotionelle de Max Scheler ${ }^{25}$. Le Bien Suprême est "vu" dans la hiérarchie des valeurs de telle manière, que sur ce terrain il n'est pas permis d'appliquer la conclusion d'induction, mais le critérium appliqué à l'appréciation de chaque bien individuel que nous découvrons en nous. Cette interprétation du texte cité du De Trinitate, est liée avant tout à la théorie de l'interieurisme augustinien, proclamée par Sciacca. C'est pourquoi probablement, quand saint Augustin a dit "si n'était imprimée en nous la notion du bien même", il n'avait pas dans sa pensée le Bien Suprême, Dieu. S'il parlait du Summum Bonum, alors, i'entière argumentation, serait superflue. Dans quel but rationnel prouver l'existence de Dieu, si d'après lui la conception est innée pour l'esprit de l'homme? ${ }^{26}$. Saint Augustin veut seulement dire, que l'essence du bien, nous la connaissons dans notre esprit et notre coeur (in interiore), c'est pourquoi nous classifions les valeurs vues dans le monde (ad extra) et les apprécions de manière intuitive et certaine. (Quand nous regardons les valeurs contingentes, c'est en même temps le Summum Bonum en tant que source de tout bien que nôus "voyons".

22 F. CAYRÉ, Dieu présent dans la vie de l'esprit, Paris, passim; Initiation à la philosophie de saint Augnstin, Paris 1947, 234-235.

23 M. F. ScIACCA, L'Existence de Dieu. Trad. par R. Jolivet, Paris 1951, passim. Voir: "Veritas habitat in interiore homine". De vera religione 39,72 PL. 34, 154; F. KöRNER, Das Prinzip der Innerlichleit in Augustins Erkenntnislehre, Würzburg 1952.

24 F. PIEMontese, La veritas agostiniana e l'agostinismo perenne, Milano 1963. Voir: De libero arbitrio 2, 3, 7 PL. 32, 1234; De Trinitate 9, 6, 9 PL. 42, 965 .

25 Voir: De Trinitate 12, 15, 25 PL. 42, 1012; Max ScHELER, Der Formalismus in der Ethik und die materialle Wertethik, Halle 1927, 262 ss.

26 La terminalogie de saint Augustin est imprécise, car le mot "ipsum bonum" une fois veut dire "bonum in genere", une autre fois "Summum Bonum" 
Saint Augustin, parlant de la connaissance intuitive du Bien Suprême, s'est inspiré des Ennéades, où Plotin très souvent a écrit au sujet

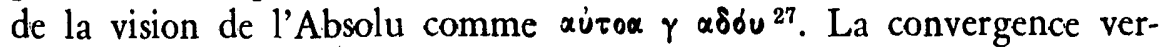
bale des énonciations de Plotin et de saint Augustin n'élimine pas la différence fondamentale, qui divise leurs systèmes. L'agnostocisme chez Plotin est plus extrême que chez saint Augustin. Ils ont aussi une conception différente de l'intuition ${ }^{28}$.

Platon et Plotin ont accepté la théorie de la imitation ( $\mu$ irnoss). Saint Augustin, expliquant la participation des créatures en Dieu, a expressèment adapté la terminologie plotinienne. Dans le Bien Suprême il voit avant tout la cause exemplaire. Elle est très caractéristique cette manière, par laquelle l'évêque d'Hippone a expliqué le bien ontinque de la nature humaine. L'âme "ne nous plaît pas par elle-même, mais par l'idée d'après laquelle elle a été faite. Car nous ne la trouvons digne d'éloges, une fois créée, que parce que nous référons à l'idéal qui a présidé à sa création" ${ }^{29}$.

Des expressions analogiques, nous en retrouvons souvent chez Plotin, qui ensuite a été fasciné par la théorie imitation de Platon ${ }^{30}$. Il faut alors se rappeler, que chez saint Augustin -comme plotinien- la relation entre le Summum Bonum et les valeurs contingentes, a été interpretée en premier lieu sur le terrain de la casualité exemplaire. L'âme humaine est seulement le bien hétéronome, c'est: pourquoi sa valeur dérive du fait, que c'est une reproduction (imitation) du Bien Primaire. Pour chaque valeur il faut parler de manière identique. Aucune créature n'est pas le bien indépendant, mais est un réflechissement du "Bien exemplaire", Dieu. Quand nous voyons le monde visible des valeurs, alors de façon intuitive nous percevons, son caractère hétéronome. A ce mo mentlà nous nous rendons compte, que chaque bien visible est seulement une reproduction ("une ombre") du Bien Suprême, qui -quoique invisible - a une existence indispensable.

Dans les considérations axiologiques de l'évêque d'Hippone il y a aussi la connaissance discursive du Bien Suprême. Souvent il est dit, que chaque bien visible -non seulement materiel, mais aussi spirituel- est de courte durée et limité dans sa perfection. "Et j’ai regardé tout le

\footnotetext{
27 Voir: Plotin, Ennéades, ed. R. Volkmann, t. II, Lipsia 1884, 460.

Voir: R.-M. Mossé-Bastide, Bergson et Plotin, Paris 1955, 108-115.

De Trinitate 8, 3, 5 PL. 42, 950, BA, t. XVI, 37.

Plotin, Ennéades 5, 1, 4.
} 
reste des choses au-dessous de toi, et j'ai qu'on ne peut pas dire, ni absolument qu'elles sont, ni absolument qu'elles ne sont pas: $[\ldots]$ car ce qui est vraiment, c'est ce qui demeure.immuablement" ${ }^{31}$. Ces traits caractéristiques prouvent, que les valeurs visibles sont toujours derivées et secondaires. Saint Augustin dans le De Trinitate a écrit en raccourci: "Il n'y aurait donc pas de biens changeants, s'il n'y avait pas un bien immuable" 32 . La conjonction causative "si" démontre, qu'ici il s'agit du résumé du raisonnement. Chaque bien inconsistant (bonum mutabile) est la confusion de la perfection, du défaut et du néant. Cette valeur n'est pas certainement une valeur première, mais une valeur secondaire. Le bien dérivé ne peut exister qu'à l'instant, où il reçoit l'existence de la cause extérieure (causa operativa). Cette cause, comme source de tout bien, est le Bien Premier et Suprême. Si aiors, dans la nature de toutes valeurs visibles il y a la mutabilité continuelle, alors il faut conclure, qu'existe aussi la Valeur Souveraine en tant que source de chaque bien. Ici nous voyons aussi l'influence de la théorie platonicienne de la participation, qui a parlé des choses comme "des copies".

Il faut aussi savoir, que saint Augustin —prouvant l'existence du Bien Suprême - a passé sous silence la problématique de l'être (ens). De la mutabilité des valeurs terrestres il y a conclu inmédiatement la nécessité de l'existence de la Valeur immuable et autonome. L'absence du probième de l'être dans l'argumentation augustinienne se joint avec l'essencialisme de la philosophie de Platon et de Plotin.

L'évêque d'Hippone fait cependant remarquer le fait, que la connaissance intuitive du Bien Suprême n'est possible que quand l'homme a des valeurs morales suffisantes. Cette remarque n'est pas une partie de l'argumentation, mais a une grande importance du point de vue psychologique. En effet une recherche intellectuelle du Bien Suprême serait menée avec succès seulement, quand l'homme intérieurement ne serait pas l'esclave des valeurs visibles. Plotin a souvent écrit sur cette affaire $^{33}$.

L'argument axiologique chez saint Augustin, a un caractère éudé-

31 Confessiones 7, 11, 17 BA, t. XIII, 619, PL. 32, 742. Voir: Enar. in ps. 127, 15 PL. 37, 1686.

32 "Quapropter nulla essent mutabilia bona, nisi essent incommutabile bonum". (De Trinitate 8, 3, 5 PL. 42, 950). Voir: A. Trapé, La nozione del muta bile e dell'immutabile secondo Sant'Agostimo, Tolentino 1959.

33 Voir: Plotin, Ennéade8, 1, 6, 7. Voir: M. F. Scracca, Saint Augustin et le néoplatonisme, Paris 1956. 
mologique. L'introduction ainsi que la fin parlent de l'amour. L'évêque d'Hippone a fini son argumentation ainsi: "Et si l'on s'attache à lui par amour, du même coup on trouve le bonheur". L'intellect parle de l'existence du Bien Suprême, mais seulement l'amour rend présent ce Bien à l'âme humaine.

Lublin, Pologne.

L'abbé Stanislas KowalczyK 